\title{
Strength distribution of solar magnetic fields in photospheric quiet Sun regions
}

\author{
J. C. Ramírez Vélez ${ }^{1}$, A. López Ariste ${ }^{2}$, and M. Semel ${ }^{1}$
}

1 LESIA, Observatoire de Paris Meudon, 92195 Meudon, France

e-mail: [Julio.Ramirez;Meir.Semel]@obspm.fr

2 THEMIS, CNRS UPS 853, c/vía Láctea s/n. 38200, La Laguna, Tenerife, Spain

e-mail: arturo@themis.iac.es

Received 11 September 2007 / Accepted 5 June 2008

\section{ABSTRACT}

\begin{abstract}
Context. The magnetic topology of the solar photosphere in its quietest regions is hidden by the difficulties to disentangle magnetic flux through the resolution element from the field strength of unresolved structures. The observation of spectral lines with strong coupling with hyperfine structure, like the observed Mn I line at $553.7 \mathrm{~nm}$, allows such differentiation.

Aims. To analyse the distribution of field strengths in the network and intranetwork of the solar photosphere through inversion of the Mn I line at $553.7 \mathrm{~nm}$.

Methods. An inversion code for the magnetic field using the principal component analysis $(P C A)$ has been developed. Statistical tests are run on the code to validate it. The code has to draw information from the small-amplitude spectral feature appearing in the core of the Stokes $V$ profile of the observed line for field strengths below a certain threshold, coinciding with lower limit of the Paschen-Back effect in the fine structure of the involved atomic levels.

Results. The inversion of the observed profiles, using the circular polarisation $(V)$ and the intensity $(I)$, shows the presence of magnetic fields strengths in a range from 0 to $2 \mathrm{kG}$, with predominant weak strength values. Mixed regions with mean strength field values of 1130 and 435 Gauss are found associated with the network and intranetwork, respectively.

Conclusions. The Mn I line at $553 \mathrm{~nm}$ probes the field strength distribution in the quiet sun and shows the predominance of weak, hectoGauss fields in the intranetwork, and strong, kiloGauss fields in the network. It also shows that both network and intranetwork are to be understood at our present spatial resolutions as field distributions, of which we hint at the mean properties.
\end{abstract}

Key words. Sun: magnetic fields - Sun: photosphere - line: profiles - methods: data analysis

\section{Introduction}

The absence of observables of the magnetism of the quiet sun is the main handicap in the determination of the magnetic topology of this region covering most of the solar photosphere. Overinterpretation of the scarce information contained in the usually observed spectral lines in magnetometry, and its comparison with numerical simulations of magnetoconvection, has been the subject of long disputes about the nature of those fields. The addition of further observables, wich were able to constrain the models and to unveil hidden ambiguities and biases in the diagnostic techniques, was mandatory in such a situation. This work is a contribution in that direction, with a further step forward in the use of Mn lines with a strong hyperfine coupling.

In a short descriptive summary of the previous studies of magnetism in quiet Sun regions, we find from one side, the Fe lines in the visible region whose circular polarisation amplitudes are almost uniquely sensitive to magnetic flux, (e.g., Keller et al. 1994; Sánchez Almeida \& Lites 2000; Lites 2002; Domínguez Cerdeña et al. 2006a; Orozco Suárez et al. 2007), and on the other side, we find the inversions of the Fe lines in the near-IR domain whose profiles are almost universally split by Zeeman effect (Lin 1995; Lin \& Rimmele 1999; Khomenko et al. 2003). Traditionally, studies based on the visible spectral lines concluded in the presence of fields with $\mathrm{kG}$ strengths and small surface coverage, while studies based on infrared lines favoured weaker hG fields and more spread in surface.
Recently, the picture has been made more realistic by considering a continuum distribution function for magnetic field strengths at each resolution element in the quiet sun (Socas Navarro \& Sánchez Almeida 2003), instead of a single magnetic vector value. Additionally, a very interesting new approach has enriched the individual line analysis in quiet regions: the inversions of simultaneous and co-spatial observations of the Fe lines in the visible $(630 \mathrm{~nm})$ and in the near-IR $(1.5 \mu \mathrm{m})$. Two such studies have already been carried out finding divergent results. In the first of these works, Domínguez Cerdeña et al. (2006a) have employed a model with three magnetic components (MISMAS model, Sánchez Almeida \& Landi Degl'Innocenti 1996) to simultaneously invert the lines in both spectral ranges. The authors retrieved field distributions in the second and third magnetic components with peaks in the strong regime and they conclude that the $\mathrm{kG}$ fields contribution dominates the magnetic flux and energy transport. In the other of these studies, Martínez González et al. (2008) showed that when only the inversions of the Fe lines in the visible are considered, the magnetic distribution is dominated by strong strength fields $(\mathrm{kG})$. However, when simultaneously near-IR and visible inversions were both considered, the $\mathrm{kG}$ contributions disappears and the distributions are mostly dominated by weak strength fields $(\mathrm{hG})$. This last conclusion is in accord with the results obtained from near-IR inversions alone (see previous references). These results, suggesting a probable bias of the quiet sun $\mathrm{Fe}$ inversions when performed 
only in the visible at $630 \mathrm{~nm}$ had also been found on numerical tests (Bellot Rubio \& Collados 2003; Martínez González et al. 2006). On the other hand, some authors have argued a possible bias in the inversions of the IR alone, suggesting that these lines are slightly sensitive to the strong strength fields, such that when mixed and not resolved magnetic structures with respective strengths of $\mathrm{hG}$ and $\mathrm{kG}$ are both present, the inferred strengths of hG are preferred over those of kG (e.g., Sánchez Almeida \& Lites 2000; Domínguez Cerdeña et al. 2006a). Such discussions and differing results illustrate the difficulty and ambiguity of the measurements attempted, and have prompted the search for more observables.

Apart from those Zeeman-based techniques discussed above, the description should be completed with the Hanle effect diagnostics, pioneered by Stenflo (1982). A paradigm of not structured and turbulent fields, e.g. Manso Sainz et al. (2004), could result in apparent contradiction to the tube-like structures used to describe Zeeman-based observations, if one insists on the picture of a single vector magnetic field per resolution element.

Mn lines subject to strong hyperfine coupling appeared as an interesting observable to be added to the previous ones: their amplitudes are dependent on magnetic flux as the usual Fe lines in the visible regime, but under certain magnetic field regimes a new spectral feature appears in the Mn profile. Just the presence or absence of such a spectral feature allows the observer to determine the presence or absence of fields with strengths below a certain threshold thus adding a new observable to the field strength to be compared to measurements with near-IR lines.

Quiet sun conditions limit the information on magnetic fields to circular polarisation profiles, whose amplitudes stay too near to the usual signal-to-noise levels found in solar polarimetry. The amplitudes of the linear polarisation Stokes parameters use to be below the noise level at the typical spatial resolutions, and are thus useless for the purpose of analysis; and, intensity profiles are insensitive to the weak magnetic fluxes involved. Consequently, one does not only need to add further spectral lines to the amount of observables, but also do it under observing conditions that guarantee very high signal-to-noise levels in polarimetry. We used the telescope THEMIS ${ }^{1}$ for that purpose in this work and we explored the capabilities of the Mn lines to provide further information on the quiet sun magnetic strength distribution. We simplified the formation of the Mn lines with a radiative transfer model using a Milne-Eddington atmosphere; the model proves to be at the limit of present possibilities with yet too many of its parameters left undetermined by the available observables. We, nevertheless, prove through synthetic tests that we can ascertain the distribution of field strengths in strong and weak flux regions (photospheric network and intranetwork respectively) with good accuracy, within the limits of the adopted Milne-Eddington modelling for the Sun.

With the results presented here, wich show a field strength distribution with maximum number of occurrences at very weak fields (less than or around $100 \mathrm{G}$ ), we propose in the conclusions a cartoon scenario that would be consistent with both the Zeeman-based measurements (visible and near-IR) and the Hanle measurements.

In Sect. 2, we review the signatures of the magnetic field strength in the line formation of the Mn I profiles. In Sect. 3, we describe the inversion code used in this work to retrieve the strength and magnetic flux from the data, and we test its capabilities with realistic noise conditions. The observations

1 Télescope héliographique pour l'étude du magnétisme et des instabilités solaires. are described in Sect. 4 and the results presented in Sect. 5. Conclusions and a final overview are included in the final section.

\section{Hyperfine structure and the Stokes parameters}

The coupling of the nuclear angular momentum $(I)$ with the orbital total angular momentum $\left(\boldsymbol{J}_{\mathrm{LS}}\right)$ gives rise to hyperfine structure (HFS). The total angular momentum $\boldsymbol{F}=\boldsymbol{J}+\boldsymbol{I}$ results in new non-degenerated atomic levels. The apparent splitting of the atomic transitions due to this effect, and the consequent broadening of solar and stellar spectral lines has been discussed in several papers since the work of Abt (1952), and used to measure the abundances of different elements (Kurucz 1993).

The most common observable for the analysis of (solar or stellar) spectral lines modified by hyperfine structure is the broadening of the line profile. Such broadening, however, can be the result of others physical phenomena as well: microturbulence, Doppler broadening, or just blended lines. Too often the ambiguity on the origin of an observed line broadening cannot be solved for, even in observations with very high spectral resolution, unless full spectropolarimetry is performed. Such is the case presented in this work, the Mn I line at $553.7 \mathrm{~nm}$ : although producing a slightly asymmetric intensity profile, the recognition of the HFS features becomes straightforward in circular polarisation, since the HFS together with an atmosphere permeated of an external magnetic field produce a unique spectral signature in the Stokes $V$ profiles. The use of this signature and its variations under different conditions as a tool for the detection of magnetic fields in the quiet sun in different field strength regimes was first proposed theoretically in López Ariste et al. (2002). Later, in López Ariste et al. (2006), the authors showed that observationally it is possible to detect the HFS effect in the circular polarisation profiles. In this work, we extend previous studies and we build an inversion code, drawing its information from those spectral features in the Stokes profiles due to HFS. The inversion algorithm is firstly validated and characterised with synthetic data, and then applied to observed data. A quantitative analysis of the field strength distribution in the quiet sun results.

For illustration and reference, we show in Fig. 1 the calculated spectral signature of the Stokes $V$ profiles for the Mn I at $553.7 \mathrm{~nm}$, as a function of the magnetic field strength. The important remark from the $V$ profiles pictured is that the weaker the magnetic field strength, the higher the amplitude of the spectral feature due to the HFS, visible in the central part of the profile.

\section{The inversion code}

\subsection{Solution to the radiative transfer equations}

The four coupled equations for the radiative transfer of the Stokes parameters were solved analytically by the code DIAGONAL (López Ariste \& Semel 1999), which was slightly modified from its original version to include the computation of hyperfine structure.

To use the code DIAGONAL we assume a Milne-Eddington atmosphere, with the possibility of including numerically gradients with optical depth of the velocity and magnetic fields, and of adding up to fifteen free parameters. Evidently, the use of a larger number of free parameters implies a higher risk of inversion ambiguities and biases, if they happen to exceed the dimensionality of the observables. But, on the other hand, considering fewer parameters may not allow to cover all the variety of the observed 

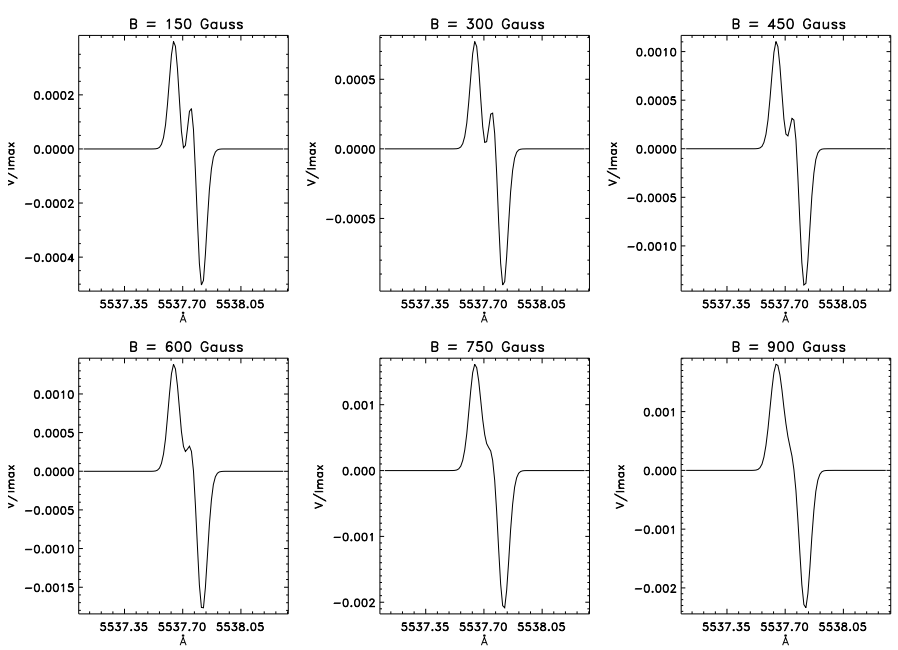

Fig. 1. Stokes $V$ profiles as a function of the magnetic field strength. Note that the spectral feature on the central part of the profiles is the signature associated to the HFS regime, and that it disappears about $B \sim$ 750 Gauss, as the line passes into the incomplete Paschen-Back regime.

profiles. We decided thus to limit the number of parameters for each atmospheric model, to the ones described below, leaving out the velocity and magnetic gradients along the line of sight.

The inversion code works in hierarchical mode by a double pass fit. In the first pass only the intensity is fitted, permitting half of the atmospheric parameters (Doppler width, $V_{\mathrm{D}}$, velocity in the line of sight $V_{\mathrm{LOS}}, \eta_{0}$, and the source function gradient, $\nabla \tau)$ to be constrained in their ranges of variation. The remaining four atmospheric parameters are related to the presence of a magnetic field: the angle between the magnetic field direction and the direction of the line of sight $\theta_{\mathrm{BLOS}}$, the azimuthal angle $\theta_{z}$, the strength of the magnetic field $B$, and a filling factor $f f$, accounting for unresolved structure. In the second (and final) fit, the eight parameters are considered in fitting simultaneously the profiles of intensity $(I)$ and circular polarisation $(V)$. Each atmospheric parameter will be left to vary randomly in the range of values specified in Table 1 to build a database for the inversions.

\subsection{Atmospheric models and database}

Let $\chi$ denote a given combination of the described atmospheric parameters such that,

$\chi_{m}=\left(V_{\mathrm{D}}, V_{\mathrm{LOS}}, \eta_{0}, \nabla \tau, B, \theta_{\mathrm{BLOS}}, \theta_{z}, f f\right)_{m}$

is the $m$ th combination. Let $\boldsymbol{S}_{m}\left(\chi_{m}\right)$ denote the Stokes vector calculated for this combination of atmospheric parameters $\chi_{m}$ :

$S_{m}\left(\chi_{m}\right)=\left(I_{m}\left(\chi_{m}\right), Q_{m}\left(\chi_{m}\right), U_{m}\left(\chi_{m}\right), V_{m}\left(\chi_{m}\right)\right)$,

and let $P_{m}$ denote any of the components of $\boldsymbol{S}_{m}\left(\chi_{m}\right)$.

In the computation Stokes parameters we have considered 201 spectral wavelength points with a step size of $12 \mathrm{~m} \AA$, covering in total $2.41 \AA$ around the line centre at $553.762 \mathrm{~nm}$. In the following, we proceed to consider each profile $P_{m}$ as a vector of 201 points $\boldsymbol{P}_{m}$. The creation of the database is the most important step for the inversions working with principal components analysis (e.g., Rees et al. 2000; Socas-Navarro et al. 2001). It is required to consider a large enough ${ }^{1}$ number of profiles in order to consider the database as statistically representative. Thus, first, we have created a database of $80802^{2}$ profiles $\boldsymbol{P}_{m}$, hereafter called the profile space matrix $(\mathcal{M})$, and we assume that all possible observed profiles in the data set are represented in the database. This assumption is however verified in the tests presented at the end of this section.

We next perform a Singular Value Decomposition (SVD) (Golub \& Van Loan 1996) of the space matrix to compute an algebraic basis of the space spanned by the profiles. We should refer to the elements of the basis as eigenprofiles.

\subsection{Analysis with PCA}

Following the synthetic notation of Eq. (2), for each one of the Stokes parameters $(I, Q, U, V)$ it is possible to construct the $201 \times$ 80802 profile matrix $\mathcal{M}$ that contains all the profiles $\boldsymbol{P}_{m}$ of the database:

$\mathcal{M}=\left(\begin{array}{lll}\boldsymbol{P}_{0} & \cdots & \boldsymbol{P}_{80801}\end{array}\right)^{\mathrm{T}}$.

After computation of the eigenprofiles, each profile in the matrix $\mathcal{M}$ can be reconstructed as:

$\boldsymbol{P}_{m}\left(\chi_{m}\right)=\Sigma_{i} \alpha_{i}\left(\chi_{m}\right) \hat{\boldsymbol{e}}_{i} ; \quad i=0,1, \ldots, 200$

where $\hat{\boldsymbol{e}}_{i}$ are the eigenvectors associated to the space profile $(\mathcal{M})$ and $\alpha_{i}$ are the coefficients of the linear combination associated to the profile $\boldsymbol{P}_{\boldsymbol{m}}$. Since the eigenvectors form an orthonormal basis of the space $(\mathcal{M})$ arranged in order of importance, one can cut the expansion in Eq. (4) and preserve only the first eigenvectors that contain relevant information on the shape of the profile. The rest of eigenvectors are associated with the noise and there is no interest in preserving them (Hansen 1992; Rees et al. 2000). In our study, we find that the appropriate number of components in Eq. (4), is $i_{\max }=30$.

With the help of the set of eigenvectors, $\left\{\hat{\boldsymbol{e}}_{\boldsymbol{i}}\right\}$, we create a second database $\left(\mathcal{M}^{\prime}\right)$ that is independent from the $(\mathcal{M})$ matrix used to compute the $\left\{\hat{\boldsymbol{e}}_{i}\right\}$. The $\left(\mathcal{M}^{\prime}\right)$ database will be decomposed in terms of the coefficients $\alpha_{i}$ as follows. Let us denote $\boldsymbol{P}_{n}$ a vector of the new (second) database. Using the Eq. (4),

$\alpha_{i}\left(\chi_{n}\right)=\boldsymbol{P}_{n}\left(\chi_{n}\right) \cdot \hat{\boldsymbol{e}}_{i} ; \quad i=0,1, \ldots, 29$.

In this way, each profile $\boldsymbol{P}_{n}$ (observed or calculated) has associated a unique set of coefficients $\left(\alpha_{0}, \ldots, \alpha_{i \max }\right)$, denoted hereafter $\boldsymbol{\alpha}_{n} \equiv\left(\alpha_{0}, \ldots, \alpha_{i \max }\right)$. So instead of working in the profile matrix space $\left(\mathcal{M}^{\prime}\right)$ it is equivalent, and faster, to work in the coefficient's space $(C)$. From Eq. (3), and in analogy with $\left(\mathcal{M}^{\prime}\right)$, we obtain for each profile $\boldsymbol{P}_{n}$ the correspondent coefficients $\boldsymbol{\alpha}_{n}$ to build the $30 \times 80802$ coefficient matrix $(C)$,

$C=\left(\begin{array}{lll}\boldsymbol{\alpha}_{0} & \cdots & \boldsymbol{\alpha}_{80801}\end{array}\right)^{\mathrm{T}}$.

\subsection{Description and validation of the inversion code}

One important quality of the code developed is that it is very easy to estimate the error bars per model parameter. We present here the tests applied for that purpose before pursuing the inversion of the observed profiles.

Let $\boldsymbol{P}_{\text {syn }}$ denote a synthetic profile with associated coefficients $\boldsymbol{\alpha}_{\text {syn }}$. The profile $\boldsymbol{P}_{\text {inv }}$, considered to be the solution by the inversion code, is found in the space of coefficients $(C)$ as the nearest set of coefficients (in an Euclidean sense) to $\boldsymbol{\alpha}_{\text {syn }}$ :

$\left\|\boldsymbol{\alpha}_{\mathrm{inv}}-\boldsymbol{\alpha}_{\mathrm{syn}}\right\|=\min \left(\left\|\boldsymbol{\alpha}_{\mathrm{syn}}-\boldsymbol{\alpha}_{n}\right\|\right) ; n=0,1, \ldots, 80801$.

2 The actual figure has no particular meaning other than, illustrating what we consider as large enough, and for future reference. 
Table 1. All the parameters vary randomly in the indicated ranges.

\begin{tabular}{lcccccccc}
\hline \hline Parameters & $V_{\mathrm{D}}(\mathrm{mA})$ & $\eta_{0}$ & $\nabla \tau$ & $V_{\mathrm{LOS}}\left(\mathrm{km} \mathrm{s}^{-1}\right)$ & $B(\mathrm{G})$ & $\theta_{\mathrm{BLOS}}(\mathrm{deg})$ & $\theta_{z}(\mathrm{deg})$ & $f f$ \\
\hline Initial Set & {$[35,75]$} & {$[0,2.5]$} & {$[0,15]$} & {$[-4,4]$} & {$[0,2000]$} & {$[0,90]$} & {$[0,90]$} & {$[0,1]$} \\
Inverted (max,min) & $(41,60)$ & $(0.7,1.8)$ & $(1.0,14.9)$ & $(0,0.54)$ & - & - & - & - \\
Mean value & 47 & 1.16 & 8.8 & 0.02 & - & - & - & - \\
\hline Final Set & {$[39,61]$} & {$[0.5,2]$} & {$[0,15]$} & {$[-2,2]$} & {$[0,2000]$} & {$[0,90]$} & {$[0,90]$} & {$[0,1]$} \\
\hline
\end{tabular}

Since each one of the Stokes parameters would give, if considered separately, one solution to the Eq. (7), let us say $\left(\boldsymbol{\alpha}_{\mathrm{inv}, I}, \boldsymbol{\alpha}_{\mathrm{inv}, Q}, \boldsymbol{\alpha}_{\mathrm{inv}, U}, \boldsymbol{\alpha}_{\mathrm{inv}, V}\right)$, it is required to find the best solution for the four Stokes parameters simultaneously. For this purpose, and given that it is a well-known fact, but particularly true in our data sets, that $I_{n} \gg V_{n}>U_{n}, Q_{n}$ necessarily implying that, in average, $\boldsymbol{\alpha}_{\mathrm{inv}, I}>\boldsymbol{\alpha}_{\mathrm{inv}, V}>\boldsymbol{\alpha}_{\mathrm{inv}, U}, \boldsymbol{\alpha}_{\mathrm{inv}, Q}$ (e.g., López Ariste \& Casini 2002), we find it useful to introduce a weight for each solution. Thanks to this, the polarised signals are weighted similarly to the intensity one, despite their disparate amplitudes.

Let the scalars

$\alpha_{w, S}=\frac{\max \left(\boldsymbol{\alpha}_{\mathrm{syn}, I}\right)}{\max \left(\boldsymbol{\alpha}_{\mathrm{syn}, S}\right)} ; \quad S=I, Q, U, V$

be the factors that modify the computation of the norms in Eq. (7), such that each one of the four solutions has the appropriate weight and can be added to the others in a linear way. The final solution, $\boldsymbol{\alpha}_{\text {sol }}$, is then found as:

$$
\left(\Sigma_{S} \alpha_{w, S}\left\|\boldsymbol{\alpha}_{\mathrm{inv}, \mathrm{S}}-\boldsymbol{\alpha}_{\mathrm{syn}, \mathrm{S}}\right\|\right)=\min \left(\Sigma_{S} \alpha_{w, S}\left\|\boldsymbol{\alpha}_{\mathrm{syn}, \mathrm{S}}-\boldsymbol{\alpha}_{n, S}\right\|\right)
$$

where $S$ denotes, as usual, the Stokes parameters $(I, Q, U, V)$.

Since we know from observations, described in Sect. 4 , that the signal-to-noise ratio in linear polarisation (Stokes $Q, U$ ) is not big enough to include them in the analysis, they were excluded as well from the tests applied to the code and only the intensity and circular polarisation were considered. This implies that from Eq. (8), $S=I, V$.

Finally, we compare the solution values of $\chi_{\text {sol }}$ (associated to $\boldsymbol{\alpha}_{\text {sol }}$ ) to the known values of $\boldsymbol{\chi}_{\text {syn }}$ (associated to $\boldsymbol{\alpha}_{\text {syn }}$ ). This comparison leads to the statistical determination of the inversion errors per model parameter, where by error we mean the absolute difference between the original and the retrieved values.

\subsubsection{Discussion of the tests}

In order to not bias the tests, we have constrained the thermodynamic parameters to the ranges of variation retrieved from the observational data sets after the first inversion (see the first three rows of Table 1). Otherwise, we have not imposed any a priori constraint to the magnetic parameters, and the profiles used in the tests were synthesized using independent uniform distributions for each parameter, following the values found in the last row of Table 1. However, in the search of direct translation to the inversion of quiet sun profiles, we have conditioned the profiles to have amplitudes in the $V$ profiles that do not exceed the maximum amplitude found in the data set $\left(V<5 \times 10^{-3}\right)$, while being bigger than the lower noise level $\left(V>5 \times 10^{-5}\right)$. Under these conditions, it is expected that the sample of profiles used in the tests is comparable with that from quiet sun data.

In Fig. 2, we show the results of the inversion of samples of 500 profiles. As a first test, we have not included noise in the inverted profiles. With the exceptions of $\nabla \tau$ and $\theta_{z}$, the errors in most of the parameters decrease rapidly to zero.
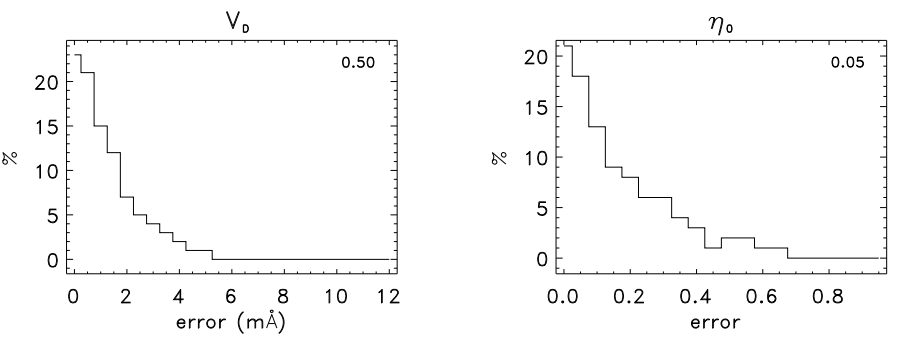

$\nabla T$

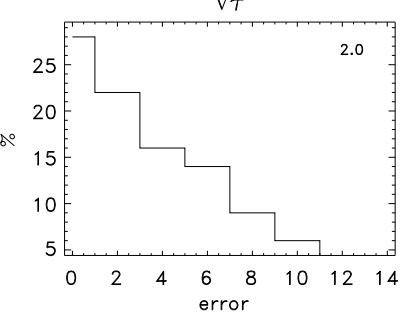

$\mathrm{B}$

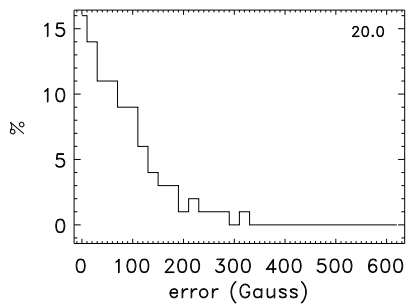

$\theta$
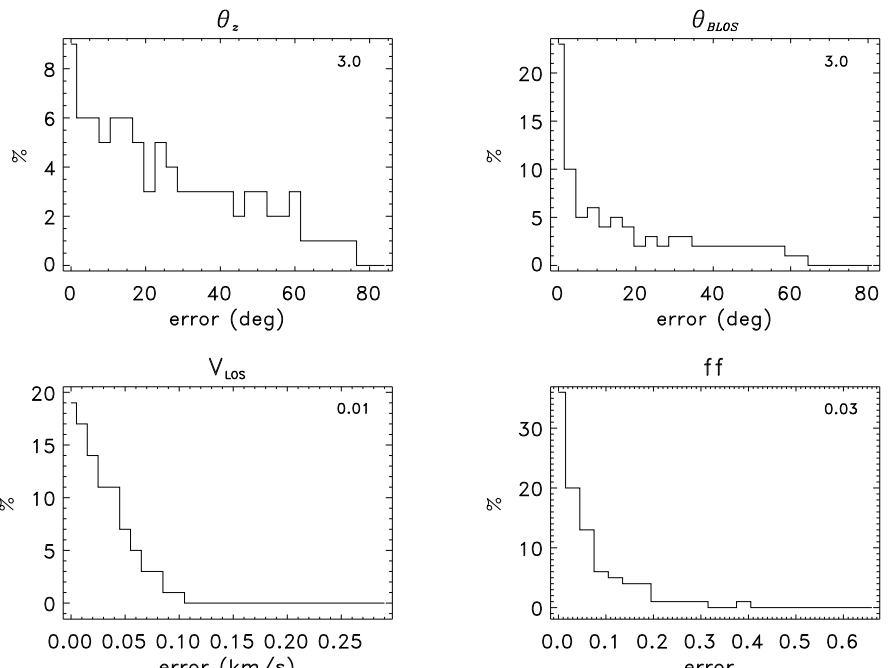

$f f$

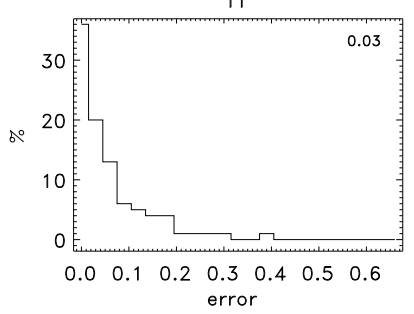

Fig. 2. Inversion histograms of each atmospheric parameter. The number in the upper right corner indicates the value of the bin size used.

The bad results for $\theta_{z}$ are due to the fact that the linear polarisation, Stokes $Q, U$, was not included in the inversions, implying that the inverted values in this parameter correspond to an aleatory distribution. Consequently, the original (by construction) and the inverted distributions in the azimuthal parameter are aleatory. Finally, plotting the absolute difference between these two distributions results in an histogram with a pick error centred in (around) zero and that decrease smoothly, like the one obtained for $\theta_{z}$.

Now, the most relevant parameter in our analysis is the magnetic field strength $B$, since the major interest of this work is to analyse the magnetic field in quiet sun conditions. In what follows, we focus the discussion on this parameter and we include also the results of inversion of profiles with noise. 
Table 2. Inversions efficiency of the magnetic field strength, expressed in percentage of correct inversions as function of $\Delta B$.

\begin{tabular}{cccc}
\hline \hline$\Delta B$ & \multicolumn{3}{c}{ Noise level } \\
& 0 & $5 \times 10^{-5}$ & $2 \times 10^{-4}$ \\
\hline $50 \mathrm{G}$ & $69 \%$ & $46 \%$ & $40 \%$ \\
$100 \mathrm{G}$ & $89 \%$ & $74 \%$ & $65 \%$ \\
$150 \mathrm{G}$ & $96 \%$ & $88 \%$ & $80 \%$ \\
$200 \mathrm{G}$ & $99 \%$ & $93 \%$ & $89 \%$ \\
$300 \mathrm{G}$ & $100 \%$ & $98 \%$ & $96 \%$ \\
\hline
\end{tabular}

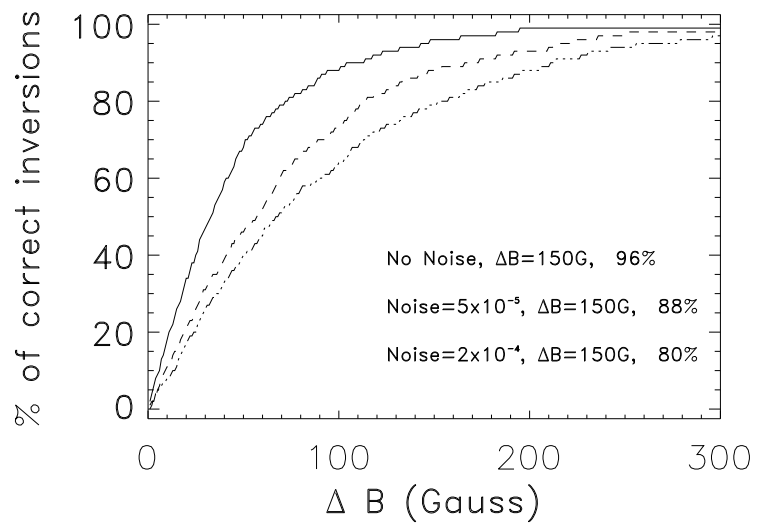

Fig. 3. Curves of cumulative percentage of correct inversions. Each sample consisted of 500 profiles. The solid line corresponds to profiles without noise. The dashed line corresponds to a noise level of $5 \times 10^{-5}$ and the dash-point-dash line to a noise level of $2 \times 10^{-4}$ the intensity of the continuum. The inset indicates the efficiency percentage for an upper limit of $\Delta B=150 \mathrm{G}$.

\subsubsection{Analysis of effective inversions in $B$}

The graphics in Fig. 2 correspond to the ideal case of profiles without noise. Considering that the real profiles have a typical noise level of the order of $2 \times 10^{-4}$ to $5 \times 10^{-5}$ the continuum level, we proceed to invert synthetic profiles with noise added to those levels. Let $B_{\text {inv }}$ denote the retrieved value of the field strength, and let $B_{\text {syn }}$ denote the real atmospheric value of the synthetic profile. Representing by $(\Delta B)$ the upper limit of the error value, such that

$\Delta B=\left|B_{\text {inv }}-B_{\text {syn }}\right|$

we can characterise the inversion efficiency behaviour of the code, understood as the percentage of profiles correctly inverted as a function of $(\Delta B)$. In Table 2 , we compare the inversions of the magnetic strength for profiles with different noise levels, and in Fig. 3 we plot the respective cumulative distribution functions.

The value of the magnetic field strength goes from 0 through $2000 \mathrm{G}$, covering all the range of magnetic strengths inherent to the quiet Sun regime. Since the spectral signature in Stokes $V$ profiles evidence of HFS regime, like those in Fig. 1, disappears for values around $B \sim 750 \mathrm{G}$, we have special interest in the behaviour of the code while inverting profiles in the weak field regime. In Table 3, we show that the precision inverting the magnetic strength is mainly independent of the magnitude of the magnetic field. The inversion gently improves with higher values of the field strength, but in general the precision in the result is preserved.

We conclude that, assuming a Milne-Eddington model, the code developed in this work for the line of Mn I at $553.7 \mathrm{~nm}$, correctly inverts profiles in very different field strength
Table 3. Inversion's efficiency percentages in the weak field regime as function of the magnetic field strength.

\begin{tabular}{cccccc}
\hline \hline$\Delta B$ & $B \leq 150 \mathrm{G}$ & $B \leq 350 \mathrm{G}$ & $B \leq 550 \mathrm{G}$ & $B \leq 750 \mathrm{G}$ & Noise \\
\hline $100 \mathrm{G}$ & $75 \%$ & $77 \%$ & $81 \%$ & $84 \%$ & No \\
$200 \mathrm{G}$ & $98 \%$ & $99 \%$ & $99 \%$ & $99 \%$ & \\
$100 \mathrm{G}$ & $60 \%$ & $57 \%$ & $61 \%$ & $64 \%$ & $5 \times 10^{-5}$ \\
$200 \mathrm{G}$ & $85 \%$ & $87 \%$ & $89 \%$ & $90 \%$ & \\
$100 \mathrm{G}$ & $56 \%$ & $53 \%$ & $54 \%$ & $57 \%$ & $2 \times 10^{-4}$ \\
$200 \mathrm{G}$ & $76 \%$ & $80 \%$ & $82 \%$ & $83 \%$ & \\
\hline
\end{tabular}

regimes: from very weak magnetic fields (lower than $100 \mathrm{G}$ ) up to $2 \mathrm{kG}$, with an error bar of $200 \mathrm{G}$ with $89 \%$ of probability (300 G with $96 \%$ of probability) independent of the field strength. With such results we can assert that, even if the actual field strength cannot be measured from inversions with sufficient precision, at least the inversion algorithm can disentangle, without any ambiguity, the $\mathrm{hG}$ from the $\mathrm{kG}$ regimes of field strength.

\section{Observations}

We carried out two campaigns of observations at the THEMIS telescope (López Ariste et al. 2000; Gelly et al. 2006) in the multiline observation mode $\mathrm{MTR}^{3}$. During the first campaign in June 2005, we searched for the spectral signature of the hyperfine structure of the MnI atom among four candidate lines in the visible spectral region at wavelengths $539.4 \mathrm{~nm}, 547.0 \mathrm{~nm}$, $551.6 \mathrm{~nm}$, and $553.7 \mathrm{~nm}$, respectively. From the data, we concluded that out of these four lines, only the one at $553.7 \mathrm{~nm}$ presented a detectable spectral signature of HFS in the Stokes $V$ profiles clear enough to be used for diagnostics. During the second campaign in June 2006 we focussed on this line. In both campaigns the Fe I doublet at 630.1 and $630.2 \mathrm{~nm}$ was observed simultaneously with the Mn I lines. Some relevant results concerning the importance of simultaneously observing spectral lines from both atomic species, as well as a brief description of the data acquisition and reduction, were presented in López Ariste et al. (2007).

In both campaigns, we scanned the solar disk centre and, in at least one day per campaign, we enjoyed excellent seeing conditions in the early morning. We have used the polarised signals levels of the Fe doublet $(630.2 \mathrm{~nm})$, observed simultaneously and co-spatially, to verify that around the scanned area there were no traces of active regions, nor enhanced magnetic areas: the Stokes $V$ amplitudes of this magnetically-sensitive line stay below $1 \%$ polarisation at all times, indicative of mostly quiet photosphere, both network and intranetwork regions alike. The data sets consisted of temporal sequences of 100 modulation cycles in polarisation. Each modulation cycle was made of 6 images with exposure times of either $300 \mathrm{~ms}$ or $500 \mathrm{~ms}$. The slit was kept fixed over the solar disk, up to residual seeing-induced image movements. In 2006, the new THEMIS correlation tracker was fully functional, thus further diminishing any residual movement. The total time of these sequences, around $8 \mathrm{~min}$, is comparable to the lifetime of the granulation. The dimensions of the slit spanned a region of 48 arcsec in two separate regions of 15.5 arcsec each, with a masked region of 17 arcsec between them (this masked region is a requirement of the polarimetry mask used for the observation, which places the splitted, orthogonal polarisation, beams 16 arcsec away one from the other in the direction along the slit). The slit width was kept at 0.5 arcsec, resulting

\footnotetext{
${ }^{3}$ Multi raie mode.
} 
in a spectral resolution of $20 \mathrm{~m} \AA$ in agreement with the pixel spectral sampling of $10 \mathrm{~m} \AA$.

We reduced the data with the DeepStokes software tool currently integrated to the THEMIS system facilities, modified to preserve spatial resolution. As delivered the DeepStokes code sums all images in the temporal series to increase the signal-tonoise ratio. Because of the long-time integration, image motion, and blurring results in a degradation of the image quality. Trying to avoid such a degradation of the spatial resolution, otherwise attained in the individual images of the time series, we modified the code so that the addition of further images in the time series was not done regardless of a further degradation in the image quality.

Specifically, in order to decide if the modulation cycle $i+1$ was to be added to the sum of the previous $i$ modulation cycles, a cut along the slit (at a pre-specified continuum wavelength of the intensity image) for the modulation cycle $i+1$ is compared to an analogous cut in the previous added $i$ cycles. The number $N$ of points which differed in both such cuts in less than $D$ was measured. When $N$ happened to be bigger than a pre-specified threshold $N_{0}$ the $i+1$ cycle was deemed spatially coherent with the previous ones and its Stokes parameters added to the series, increasing the signal-to-noise ratio. If the $i+1$ cycle failed to pass the test, it was assumed that the slit had moved and a new time series was started at point $i+1$. The sensitivity threshold $D$ and the minimum number of cases $N_{0}$ are provided by the user. We determined empirically their value from a posteriori considerations on attained signal-to-noise ratios and spatial resolutions, and the trade-off among both parameters. In the cases under analysis in this work, the achieved noise levels laid between $5 \times 10^{-5}$ and $2 \times 10^{-4}$ of the continuum level, for a spatial resolution better than 1 arc sec and approaching at times $0.6 \operatorname{arcsec}^{4}$.

\section{Results}

In Fig. 4, we show for illustration some of the observed Stokes I and $V$ profiles (dotted lines) and the correspondent fits from the inversion (solid lines) overplotted. The panel covers the diversity of magnetic field strengths found in the quiet sun as seen through the Mn I lines: from the weak intensity regime $(\mathrm{hG})$ to the strong regime $(\mathrm{kG})$.

\subsection{The complete set of profiles}

Only $14 \%$ of the total amount of data had a $\mathrm{S} / \mathrm{N}$ ratio high enough to be inverted reliably: around 500 profiles. In Fig. 5, we show the resulting magnetic field strength and longitudinal flux density distributions (defined as filling factor times the longitudinal field strength) from those inversions. For better description, we have fitted the histograms to an empirical distribution function of the form $N(x)=a \exp (-x / b)+c$, where $a, b$, and $c$ are positives constants and $\mathrm{N}(\mathrm{x})$ represents the number of cases. The introduction of a uniform distribution (the constant $c$ ) is required to fit the flat part of the histograms, corresponding to intermediate and high strength and flux density values. Otherwise, the left part of the histogram, associated with weak strength and flux density values respectively, is well fitted with an exponential function. In the case of the field strengh distribution, the fitting values are $a=672, b=51.3 \mathrm{G}$ and $c=8.6$, while in the case of the flux density $a=465, b=6.3 \mathrm{Mx} / \mathrm{cm}^{2}$ and $c=1.7$.

\footnotetext{
4 The spatial resolution was gauged by the crude and simple method of counting and measuring the size of brighter-than-average and darkerthan-average regions as visible in the continuum cuts along the slit.
}

The full widths at half maximum $(F W M H)$ are respectively $F W M H_{B}=54 \mathrm{G}$ and $F W M H_{\text {Flux }}=4.6 \mathrm{Mx} / \mathrm{cm}^{2}$, and the correspondent mean values are $\langle B\rangle=635 \mathrm{G}$ and $\langle$ Flux $\rangle=13 \mathrm{Mx} / \mathrm{cm}^{2}$.

Although one expects mostly maxwellian-like probability distribution functions (PDF) (Domínguez Cerdeña et al. 2006ab) for the field strength, because of the coarseness of the histogram bins, we prefer to fit the data with an exponential for the weak fields plus a constant distribution for the strong fields. In spite of that empirical fit, the histogram shown in Fig. 5 should not be translated into the most probable strength of the inverted profiles is zero $\mathrm{G}$, but rather into some value between $0<B<75 \mathrm{G}$, included in the lower bin of the histogram. The main information the histograms show is that the weak field strength regime $(\mathrm{hG})$ is more abundant or dominates the surface coverage in the quiet sun conditions (70\%). Nevertheless, a considerable amount of cases, $30 \%$ of the inverted profiles, present magnetic strengths superior to $1000 \mathrm{G}$, i.e., in the strong field strength regime, and cannot be considered as absent.

\subsection{Network versus intranetwork}

In order to study how the presence of network and intranetwork regions influence the results shown in the histograms of Fig. 5, we proceed to differentiate the profiles between these two regions. To this purpose, we have used the Fe I line $(5538.52 \mathrm{~nm})$, sitting next to the Mn line, to set a criterion for the classification of profiles into one of the regions. We arbitrarily set the following condition for points to belong to the photospheric network: If the level of the normalised circular polarisation $V / I$ in the $\mathrm{Fe}$ I line exceeds a threshold value $(T V)$ of the continuum level we consider the point as part of the network. All other points are considered intranetwork. We have initially used two threshold values: $T V=1 \times 10^{-3}$ and $2 \times 10^{-3}$. In Fig. 6, we show both distributions (for field strength and longitudinal flux density) for each one of the regions. The solid line represent a $T V=1 \times 10^{-3}$ and the gray dotted line is for $T V=2 \times 10^{-3}$. When using the upper threshold, $T V=2 \times 10^{-3}$ corresponding to higher flux concentrations, the contribution of profiles in the weak strength regime in the network region has almost disappeared, while the number of profiles with strong field strengths has been just slightly modified. On the other hand, the use of the moderate threshold value $1 \times 10^{-3}$ already identifies the profiles in the strong regime in the network region as well as a nonnegligible contribution of fields with weak strengths. In the intranetwork set, with either one or the other threshold, the general shape of the histogram remains unchanged. It is clear that a different threshold value able to radically change the general shapes of the histograms for both (network and intranetwork) regions would be unacceptably high and unrealistic. Therefore, and keeping in mind the small dependence of the network-assigned weak fields on the threshold value, we adopt in what follows the network and intranetwork definitions using the lower TV, with correspondent distributions function represented by the solid line in Fig. 6. To complete the justification on the criterion use for classification into network and internetwork, let us add that from previous work (López Ariste et al. 2006) we know that the described classification, however arbitrary, successfully identifies most of the network as identified visually over a magnetograph image. In terms of magnetic flux, the threshold value of $1 \times 10^{-3}$, would correspond roughly to $10 \mathrm{Mx} / \mathrm{cm}^{2}$, and it is certainly biassed towards including any flux concentration found in the intranetwork into the network, and conversely, any low-flux region in the network will be included in the intranetwork dataset, a bias that we should remember in the conclusions. 
J. C. Ramírez Vélez et al.: Strength distribution of solar magnetic fields in photospheric quiet Sun regions
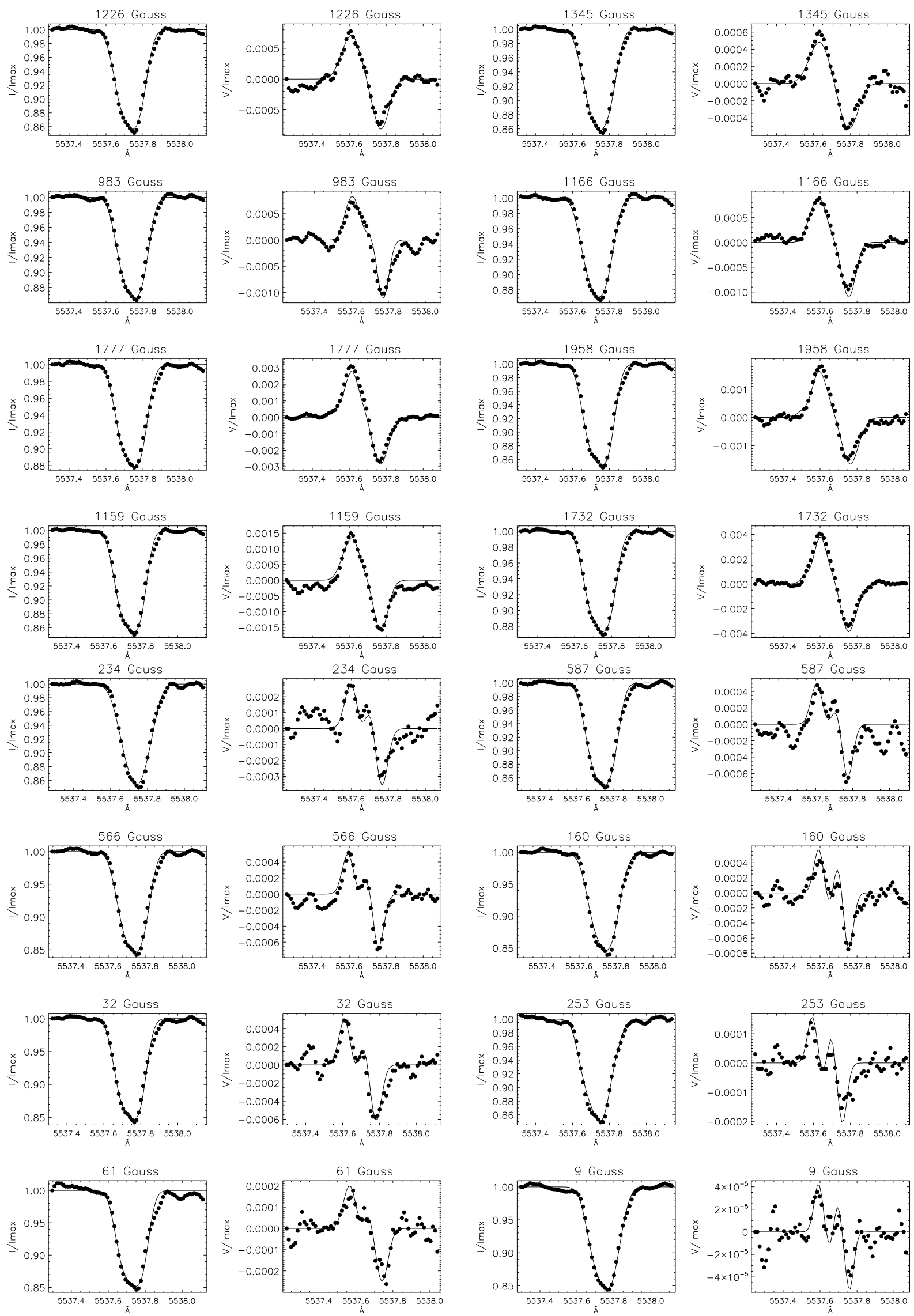

Fig. 4. Examples of inverted profiles. The dotted lines correspond to the observed data and the solid line to the respective fitted profile. The profiles were inverted without any smoothing, but in this plot a 2-pixel smoothing has been applied to observed data. 

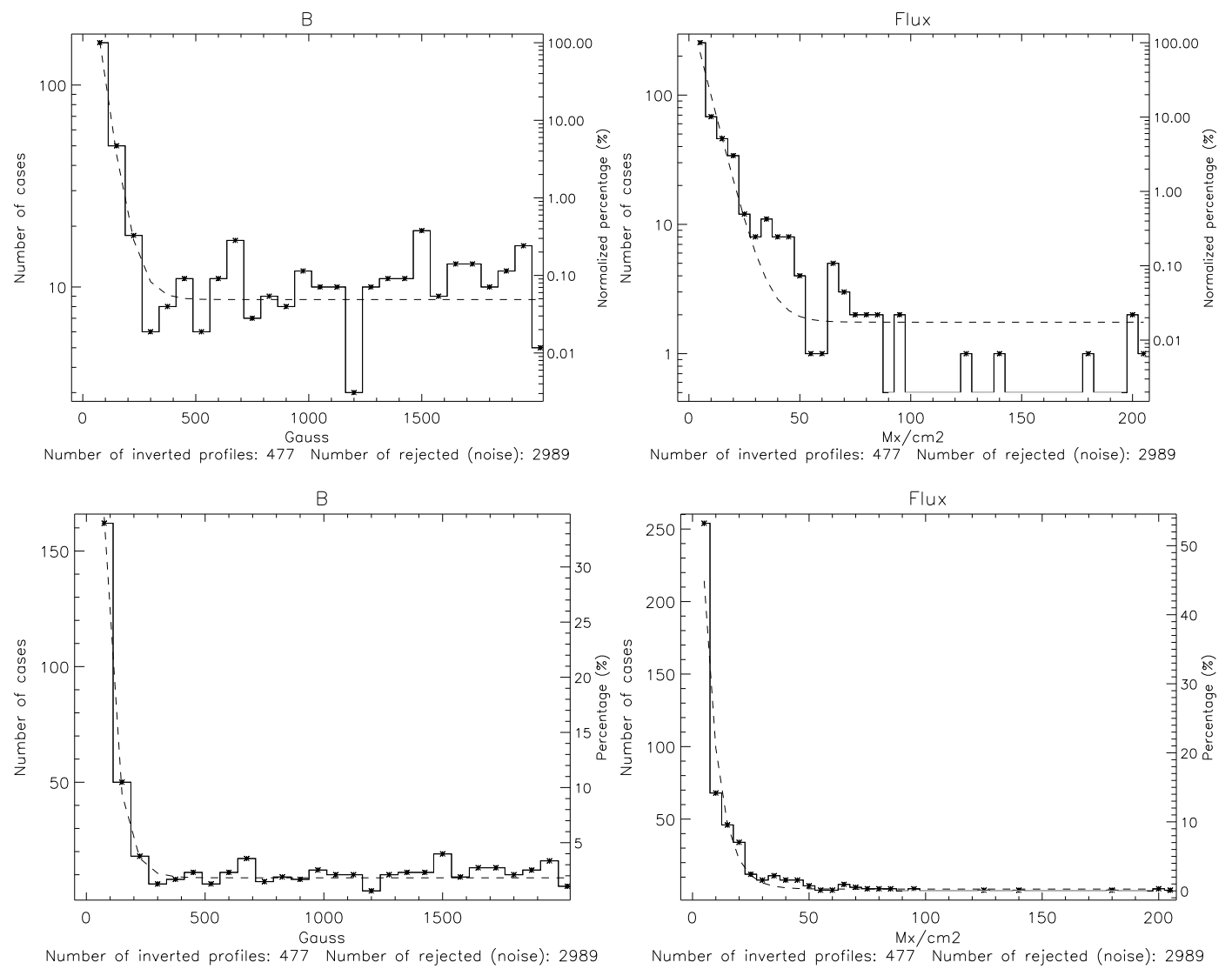

Fig. 5. Top panels: histograms of the distribution of the magnetic field strength and the longitudinal magnetic flux represented in logarithmic scale. Bottom panels: the same histograms in normal scale. The dashed lines represents the fitted distribution functions $\mathrm{N}(\mathrm{x})$. The bin sizes are $75 \mathrm{G}$ and $5 \mathrm{Mx} / \mathrm{cm}^{2}$ in field strength and flux, respectively.
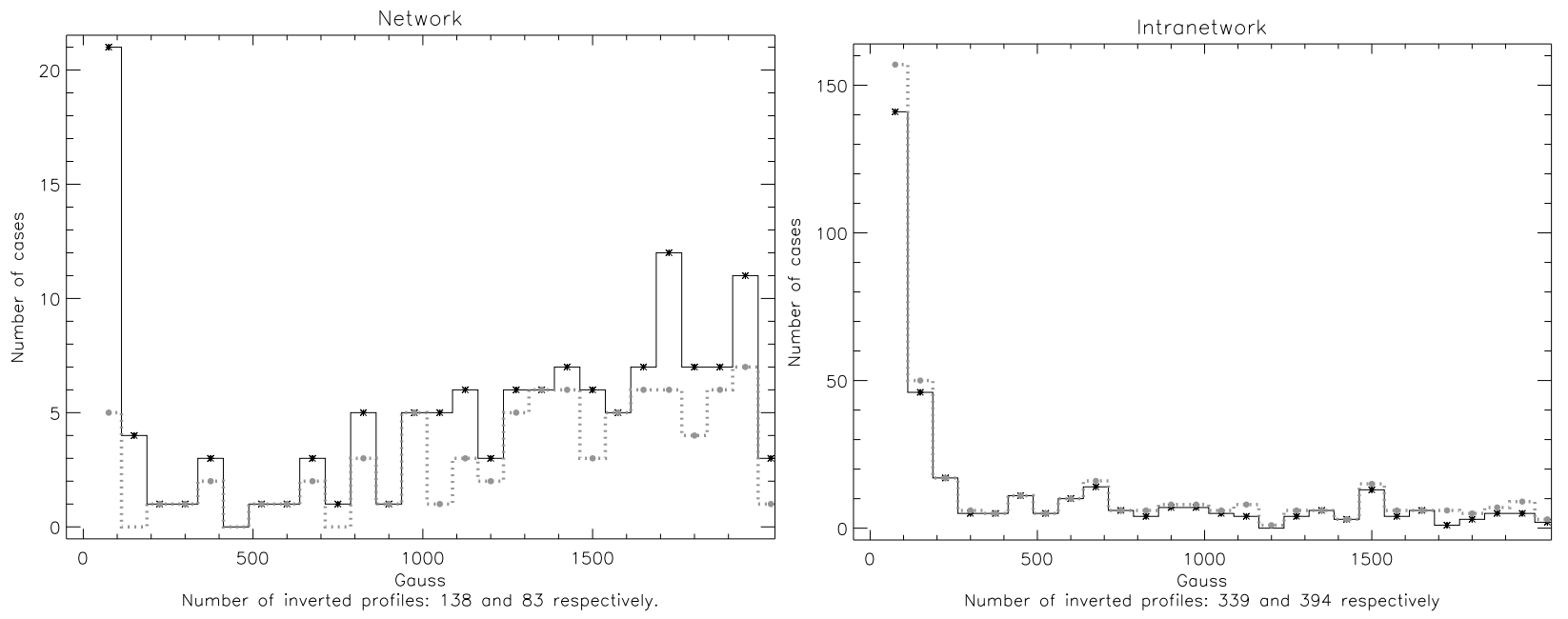

Fig. 6. Histograms of network and intranetwork regions. The solid (dotted) line correspond to a threshold value of $1 \times 10^{-3}\left(2 \times 10^{-3}\right)$. In the network, the contribution of profiles with strength superior to $1 \mathrm{kG}$ is considerably more important than in the intranetwork.

After classification, from a sample of 477 inverted cases, it was found that $70 \%$ belonged to the intranetwork region, while the remaining $30 \%$ were from the network. As already noted in the previous paragraph, the correspondent distributions for the magnetic field strength in the network region, represented by the solid line in the left panel in Fig. 6, clearly differs from the internetwork distribution and also from the complete dataset distribution: it presents an apparent growth in the number of cases in the strong field regime, in a striking bimodal distribution. On the other side, the intranetwork distribution maintains a similar shape to the one found before: the intranetwork appears to be predominantly permeated by weak fields.

The main conclusion arising from these separate distributions is that the derived magnetic strength distribution from the inversions of the $\mathrm{Mn}$ I line in the visible range at $553 \mathrm{~nm}$, in the low-flux quiet sun or intranetwork region (taking into account the known bias of our definition of intranetwork) is dominated in number of cases by weak field-strength fields. This is in accord with previous results obtained with the analysis by measurement in the infrared range (Khomenko et al. 2003; Lin 1995; 
Table 4. Comparison between the total set of inverted profiles and the Network-Intranetwork profiles.

\begin{tabular}{lccc}
\hline \hline & Total inverted & Intranetwork & Network \\
\hline Number of Profiles & 477 & 339 & 138 \\
Strong regime $(\mathrm{kG})$ & $30 \%$ & $17 \%$ & $63 \%$ \\
Weak regime $(\mathrm{hG})$ & $70 \%$ & $83 \%$ & $37 \%$ \\
$\langle B\rangle(\mathrm{G})$ & 635 & 435 & 1130 \\
$\langle$ Flux $\rangle\left(\mathrm{Mx} / \mathrm{cm}^{2}\right)$ & 12.8 & 7.3 & 26.5 \\
\hline
\end{tabular}

Lin \& Rimmele 1999), in the visible (Keller et al. 1994) and with the recently retrieved distributions from the very high spectral resolution (0.3 arcsec) HINODE data (Orozco Suárez et al. 2007). Moreover, we found that the most probable expected strength is $B<100 \mathrm{G}$, close to the result obtained from simultaneous inversions from the infrared and from the visible recently published in Martínez González et al. (2008).

On the other hand, strong flux regions, mostly the photospheric network, rather show a bimodal distribution of fields with a contribution of weak field-strength fields comparable to that found in weak-flux regions, and an increased appearance of kG fields (Domínguez Cerdeña et al. 2006ab; Lites 2002).

In Table 4, we summarise these results in terms of inversion percentages for different magnetic strengths as seen in the histograms of the analysed data sets previously discussed.

\section{Conclusions}

In this work, we contribute to the exploration of the diagnostic capabilities of Mn lines with strong hyperfine coupling for the magnetic topology of the quiet sun. We built an inversion code for the Mn line at $553.7 \mathrm{~nm}$ formed in a Milne-Eddington atmosphere, and tested it under the conditions typical of the quiet sun in terms of signal amplitudes and noise levels. Such conditions are highly constraining: in most of the cases the magnetic field information is contained exclusively in the Stokes $V$ profile, the linear polarisation signals being under noise levels and the intensity profile being dominated by the non-magnetic portion of the resolution element. Under such restrictive conditions the number of independent observables for the magnetic field is highly reduced (Asensio Ramos et al. 2007) and it sets a limit to the number of free parameters of our model. Previous works have shown that the Mn lines increased the number of observables thanks to the spectral features appearing under certain magnetic regimes. The inversion code we tested in this work takes advantage of it and allows us to improve over the simplistic weakfield approximation of previous works into a more sophisticated Milne-Eddington atmosphere and yet to conserve the possibility of disentangling strong from weak fields.

Since most of the other parameters in the Milne-Eddington model, aside from field strength and flux, were not determined accurately under quiet-sun conditions, we doubt that any more sophisticated model can be used in the diagnostics at this point without the addition of further independent observables (e.g., with other spectral lines). However, more sophisticated models can shed light into implicit biases in such unrealistic picture of the solar photosphere as a Milne-Eddington atmosphere (e.g., Sánchez Almeida et al. 2008).

We have payed special attention throughout this work to the numerical tests made to ascertain whether, under present observational conditions of the quiet sun, the weak fields were disentangled from the strong fields for similar net fluxes. The answer is affirmative, and therefore we proceeded to apply the code to real data taken with the THEMIS telescope under good seeing conditions. Even in a telescope like THEMIS, focussed on performing the most sensitive polarimetry possible, the acquisition of the data at the required signal-to-noise levels implied a tradeoff in spatial resolution (seldomly better than 1 arcsec) that limits our results. We therefore constrained ourselves to separate the photospheric network from the intranetwork in our study, and restrained ourselves to identifying smaller scales, like granules and intergranules.

To identify network from intranetwork we applied a rather poor rule fixing at $10^{-3}$ the smallest amplitude of the Stokes $V$ profile respect to the continuum intensity for a point to belong to the photospheric network. Such a rule obviously classifies as network any high flux concentration, mostly found over the network, but not totally absent from the intranetwork. Conversely, it calls intranetwork any low-flux concentrations, mostly found all over the intranetwork, but not absent from the network either. With such bias in mind we decided to keep the names of network and intranetwork for the two classes because a small change in such threshold value did not change the general shape of the histograms, but also because of the following reasons.

After inversion, the intranetwork points show a distribution of field strengths whose dominant feature can be easily fitted with an exponential tail. We use the noun tail because an exponential distribution would result in a non-zero probability for null fields, something unacceptable for a vector field. Such a distribution, showing that the quiet sun regions are mostly permeated of weak strength fields, would be in full agreement with previous results in both Hanle and Zeeman effects (Manso Sainz et al. 2004; Lin \& Rimmele 1999; Khomenko et al. 2003; Martínez González et al. 2008) and reveals a random or disorganised vector magnetic field at scales much smaller than present spatial resolution, if Hanle and Zeeman diagnostics are to be coherent with each other and with a field strength whose highest probability remains below 100 G (Sánchez Almeida et al. 2003).

The network points, on the other hand, show a bimodal distribution: the exponential tail found in the intranetwork distribution is still present, peaking at field strengths below $100 \mathrm{G}$. But on top of that disorganised magnetic regime we observe the appearance of stronger fields, in the kiloGauss regime. This second distribution of fields would be in agreement with the presence of one or several concentration mechanisms, able to stabilise, organise, and perhaps amplify the statistical fluctuations of the ubiquitous turbulent field in those places where photospheric dynamics or sheer accidents are able to maintain a temporal coherence bigger than the typical magnetic diffussivities (Parker 1982). One can heuristically think that the strong down drafting plumes in the vertex of the convection cells are such places (Nordlund et al. 1992; Rast 2003), thus defining the magnetic photospheric network as a non-continuous succession of bright network points (Muller \& Roudier 2003) where magnetic fields are organised in vertical structures and made stable by the downdrafting plasma. Other smaller plumes found in mesogranular or granular scales (Rast 2003) may also survive long enough to allow the statistical concentration of high fluxes, to be found here and there throughout the intranetwork and that, in our analysis, would have been included in the network group.

The cartoon just drawn above is in agreement with the histograms measured in the present work over the quiet sun. In particular, we are prone to the presence of an ubiquitous random field, as inferred from Hanle diagnostics, and whose statistical fluctuations can be seen as Zeeman signatures over deep magnetograms, but otherwise highly disorganised at present resolutions except when plasma movements create conditions, as around 
convection plumes, stable enough to concentrate and build up net magnetic flux. In any case, with the inversions performed here on the Mn I in the visible region of the spectra, at $553 \mathrm{~nm}$, we have found that the distributions of the strength fields in the quiet Sun regions are dominated in occurrence by weak strength fields except in those areas related with the network.

Acknowledgements. J.C.R.V. thanks to M. J. Martínez González for all helpful discussions and also to the referee, J. Sánchez Almeida, for all the comments that served to improve the final state of the manuscript.

\section{References}

Abt, A. 1952, ApJ, 115, 199

Asensio Ramos, A., Socas-Navarro, H., López Ariste, A., \& Martínez González, M. J. 2007, ApJ, 660, 1690

Bellot Rubio, L. R., \& Collados, M. 2003, A\&A, 406, 357

Domínguez Cerdeña, I., Kneer, F., \& Sánchez Almeida, J. 2003, ApJ, 582, L55 Domínguez Cerdeña, I., Sánchez Almeida, J., \& Kneer, F. 2006a, ApJ, 646, 1421

Domínguez Cerdeña, I., Sánchez Almeida, J., \& Kneer, F. 2006b, ApJ, 636, 496

Gelly, B., \& López Ariste, A. 2006, sf2a.conf, 539

Golub, G. H., \& Van Loan, C. F. 1996, Matrix Computations, 3rd edn. (Baltimore: Johns Hopkins University Press)

Hansen, P. C. 1992, SIAM Rev., 34, 561

Keller, C. U., Deubner, F.-L., Egger, U., Fleck, B., \& Povel, H. P. 1994, A\&A, 286, 626

Khomekno, E. V., Collados, M., Solanki, S., Lagg, A., \& Trujillo Bueno, J. 2003, ApJ, 115, 199

Lin, H. 1995, ApJ, 446, 421
Lin, H., \& Rimemele 1999, ApJ, 514, 448

Lites, B. W. 2000, ApJ, 573, 431

López Ariste, A., \& Casini, R. 2002, ApJ, 575, 529

López Ariste, A., \& Semel, M. 1999, A\&A, 139, 417

López Ariste, A., Rayrole, J., \& Semel, M. 2000, A\&A, 142, 137

López Ariste, A., Tomczyk, S., \& Casini, R. 2002, ApJ, 580, 519

López Ariste, A., Tomczyk, S., \& Casini, R. 2006, A\&A, 454, 663

López Ariste, A., Martínez González, M. J., \& Ramírez Vélez, J. C. 2007, A\&A, 464, 351

Manso Sainz, R., Land Degl'Innocenti, L., \& Trujillo Bueno, J. ApJ, 2004, 614, 89

Martínez González, M. J., Collados, M., \& Ruiz Cobo, B. 2006, A\&A, 456, 1159

Martínez Gonzalez, M. J., Collados, M., Ruiz Cobos, B., \& Beck, C. 2008, A\&A, 477,953

Muller, R., \& Roudier, Th., SoPh, 1992, 14127

Nordlund, A., Brandenburg, A., Jennings, R. L., et al. 1992, ApJ, 392, 647

Orozco Suárez, D., Bellot Rubio, L. R., del Toro Iniesta, J. C., et al. 2007, ApJ, 670, L61

Parker, E. N. 1982, ApJ, 256, 292

Kurucz, R. L. 1993, Phys. Scr., T47, 110

Rast, M. P. 2003, ApJ, 597, 1200

Rees, D. E., López Ariste, A., Thatcher, J., \& Semel, M. 2000, A\&A, 355, 759

Sánchez Almeida, J., \& Landi degl'Innocenti, E. 1996, Sol. Phys., 164, 203

Sánchez Almeida, J., \& Lites, B. W. 2000, ApJ, 532, 1215

Sánchez Almeida, J., Emonet, T., \& Cattaneo, F. 2003, ApJ, 585, 536

Sánchez Almeida, J., Viticchié, B., Landi Degl'Innocenti, E., \& Berrilli, F. 2008, ApJ, 675, 906

Socas Navarro, H., \& Sánchez Almeida, J. 2003, ApJ, 593, 581

Socas Navarro, H., López Ariste, A., \& Lites, B. W. 2001, ApJ, 553,949

Stenflo, J. O. 1982, SoPh, 80, 209S 